\title{
NONISOLATED FORMS OF RATIONAL TRIPLE POINT SINGULARITIES OF SURFACES AND THEIR RESOLUTIONS
}

\author{
A. ALTINTAŞ SHARLAND, G. ÇEVİK AND M. TOSUN
}

\begin{abstract}
We give a list of nonisolated hypersurface singularities of which normalizations are the rational triple point singularities of surfaces and construct their minimal resolution graphs by a method introduced by Oka for isolated complete intersection singularities. We also show that nonisolated forms of rational triple point singularities and their normalizations are both Newton non-degenerate.
\end{abstract}

1. Introduction. Let $S$ be a germ of a normal surface embedded in $\mathbb{C}^{N}$ with a singularity at the origin. Let $\pi: \widetilde{S} \rightarrow S$ be a resolution of $S$. The singularity of $S$ is called rational if $H^{1}\left(\widetilde{S}, \mathcal{O}_{\tilde{S}}\right)=0$. This condition implies very nice combinatorial results on the invariants of rational singularities obtained from their resolution graphs [2]. For example, the multiplicity of a rational singularity equals $-Z^{2}$ where $Z$ is Artin's divisor (see Section 2) of the minimal resolution graph of the singularity. The rational singularities of surfaces of multiplicity 2 are famously known as rational double or Du Val singularities (see, for example, [3]). In [2], Artin classified the minimal resolution graphs of rational singularities of surfaces of multiplicity 3 (rational triple point singularities or RTP-singularities, for short). The list of minimal resolution graphs for multiplicity 4 and 5 were given in $[\mathbf{2 3}, \mathbf{2 5}]$, respectively. Those graphs were obtained by using the combinatoric properties of the minimal resolution graphs of rational singularities.

2010 AMS Mathematics subject classification. Primary 58K20.

Keywords and phrases. Rational singularities, nonisolated hypersurfaces, Newton polygon, resolution graphs.

The second and third authors were supported by project 109T667 under program 1001 of the Scientific and Technological Research Council of Turkey.

Received by the editors on January 26, 2013, and in revised form on June 4, 2014.

DOI: $10.1216 / \mathrm{RMJ}-2016-46-2-357$

Copyright (C)2016 Rocky Mountain Mathematics Consortium 
The classification problem of rational singularities by their minimal graphs was studied in depth in $[\mathbf{1 9}, \mathbf{2 6}]$.

In [28], Tjurina proved that rational singularities of surfaces are absolutely isolated, i.e., they can be resolved by blowing up without normalization, and gave a list of explicit equations defining the RTPsingularities. Her construction is based on the fact that a subgraph of a resolution graph of a rational singularity is still a resolution graph of a rational singularity ([2]). According to [28], a surface having an RTP-singularity is defined by three equations in $\mathbb{C}^{4}$ (see also Section 4 ). So, they are not hypersurface singularities nor complete intersection singularities. This makes them one of the most interesting objects in singularity theory/algebraic geometry, as they provide examples and a better understanding of other singularities of surfaces. More generally, a rational singularity of multiplicity $m$ can be given by $m(m-1) / 2$ equations with linearly independent quadratic terms [31].

In this work, we study the equations defining RTP-singularities and give a new construction of their minimal resolution graphs. Our presentation is divided into three main sections. After recalling some facts on rational singularities of surfaces, we recall a global construction of triple covers from algebraic geometers point of view in Section 3. Then, using the fact that any normal surface singularity occurs as the normalization of a nonisolated hypersurface singularity, we obtain explicit equations of some nonisolated hypersurfaces in $\mathbb{C}^{3}$ so that their normalizations give RTP-singularities. Since the normalizations of our equations exactly correspond to the ones listed by Tjurina (see Proposition 4.2) we will call them nonisolated forms of RTP-singularities.

A list for nonisolated forms of RTP-singularities was also obtained in [5] by a different construction and some of the equations (such as $A_{k-1, \ell-1, m-1}, C_{k-1, \ell+1}$ and $\left.F_{k-1}\right)$ different from ours. Their construction is based on [27] where the author studied triple covers $Y \rightarrow X$ by global data on $X$ using the classical method of solving cubic equations and presented conditions for the cover to be smooth with smooth branch locus and other properties. In the case of surfaces, that technique provides a resolution of singularities of both the branch locus and of $Y$. This method is, in fact, called Jung's resolution of singularities, studied in $[4,15]$. 
The cubic equations of nonisolated forms of RTP-singularities listed here may not have the simplest forms, but they are obtained by suitable projections for our purposes in Section 4. There, we construct an abstract graph from an arbitrary polygon in $\mathbb{R}^{3}$ by a regular subdivision and show that it may not correspond to a resolution graph of a singularity if it is not a Newton polygon (see subsection 4.2 .2 and Remark 4.25). Then we construct the minimal resolution graphs of RTP-singularities using the Newton polygons of those cubic equations. This method is given in [24] in the case of non-degenerate complete intersection singularities. Here, we simplify the method (for example, Definition 4.22 which comes from tropical geometry), and refer to it as Oka's algorithm. Even though many results in [24] concern complete intersection singularities, some of those have the "isolated singularity" hypothesis (e.g., [24, Theorem 6.2]), and no nonisolated examples were presented there. The equations given here are the first examples in the literature of nonisolated hypersurface singularities for which Oka's algorithm works.

In the final section, we show that both normal surfaces in $\mathbb{C}^{4}$ and their nonisolated forms in $\mathbb{C}^{3}$ are non-degenerate, which means that they can be resolved by toric modifications associated with the regular subdivision of the corresponding Newton polygon. This fact was shown in [30] for isolated hypersurface singularities and generalized in [24]. We also recall a more general definition of nondegeneracy given in [1], where it was proved that all non-degenerate singularities can be resolved by toric modifications, in order to show that the nonisolated forms of RTP-singularities are non-degenerate. This interesting property leads us to ask whether a normal surface singularity is non-degenerate if and only if its nonisolated form is nondegenerate.

2. Preliminaries on rational singularities. Assume that $S$ is a normal surface with a singularity at 0 embedded in $\left(\mathbb{C}^{n}, 0\right)$ which means that the local ring $\mathcal{O}_{S, 0}$ is normal. This means that 0 is an isolated singularity. A resolution of $S$ is a proper map $\pi:(\widetilde{S}, E) \rightarrow S$ such that $\widetilde{S}$ is a nonsingular surface, $E:=\pi^{-1}(0)$ and the restriction of $\pi$ to $\pi^{-1}(S-0)$ is an isomorphism. The fibre $E$ is called the exceptional divisor of $\pi$ which is, by Zariski's main theorem ([12, 
Theorem V.5.2]), a connected curve. A resolution $\pi$ is called minimal if any other resolution of $S$ factorizes via $\pi$. The minimal resolution exists and is unique.

Note that, when $S$ has a nonisolated singularity, we first apply a normalization $n:(\bar{S}, 0) \rightarrow S$ where $\bar{S}$ is a normal surface, $n$ is a finite and proper map.

Theorem 2.1 ([32]). Any reduced complex surface admits a resolution.

Definition 2.2. A normal surface singularity $S$ is called rational if $H^{1}\left(\widetilde{S}, \mathcal{O}_{\tilde{S}}\right)=0$.

Note that this characterisation is independent of the choice of the resolution. The exceptional divisor of a rational singularity is a normal crossing. Each component $E_{i}$ of $E$ is a nonsingular rational curve, and its resolution graph is a tree (see, for example, [29]). Moreover, by [28], rational singularities can be resolved by a finite number of blowups (without normalization). We also have a combinatorial description of rational singularities.

Theorem 2.3 ([2]). A normal surface singularity $S$ is rational if and only if $p_{a}(Y) \leq 0$ for any resolution $\pi:(\widetilde{S}, E) \rightarrow(S, 0)$ where $p_{a}(Y)$ is the arithmetic genus of the positive divisor $Y:=\sum a_{i} E_{i}$, i.e., $a_{i} \geq 0$ for all $i$, supported on the exceptional divisor $E=\cup_{i=1}^{n} E_{i}$.

Moreover, among all positive divisors $Y$ supported on $E$ such that $\left(Y \cdot E_{i}\right) \leq 0$ for all $i=1, \ldots, n$, there exists a smallest divisor, called Artin's divisor of the resolution $\pi$ and denoted by $Z$. We have

$$
Y:=\sum a_{i} E_{i} \geq Y^{\prime}:=\sum a_{i}^{\prime} E_{i}
$$

if $a_{i} \geq a_{i}^{\prime}$ for all $i=1, \ldots, n$, with $a_{i} \geq 0$ and $a_{i}^{\prime} \geq 0$. One aspect of the information we can obtain from Artin's divisor is the multiplicity of the corresponding rational singularity.

The multiplicity of $S$ at 0 is defined as the number of intersection points of $S$ by a generic affine space of codimension 2 in a small 
neighbourhood of the origin. It plays a key role in the study of singularities. In the case of rational singularities, it can be obtained from the resolution.

Proposition 2.4 ([2]). Let $S$ be a rational singularity of multiplicity $m$ at 0 . Then $Z^{2}=-m$ and the embedding dimension equals $m+1$.

Remark 2.5. Conversely, if a given graph is weighted by $\left(w_{i}, g_{i}\right)$ at each vertex such that it is a tree with $g_{i}=0$ for all $i$ and satisfies $-m+\sum_{i=1}^{n} a_{i}\left(w_{i}-2\right) / 2+1=0$ (cf., Theorem 2.3 and Proposition 2.4), then it is a resolution graph of a rational singularity, and the integers $a_{i}$ are coefficients of the Artin divisor. In this case, the numbers $w_{i}$ and $g_{i}$ represent $-E_{i}^{2}$ and the genus of $E_{i}$ in the exceptional fibre, respectively.

3. Triple covers after Miranda. In [20], Miranda showed that a set of data for a triple cover $p: Y \rightarrow X$ between two algebraic schemes (over characteristic $\neq 2,3$ ) consists of a free $\mathcal{O}_{X}$-module $\mathcal{E}$ of rank 2 (say, generated by $z$ and $w$ ) such that $p_{*} \mathcal{O}_{Y} \cong \mathcal{O}_{X} \oplus \mathcal{E}$ and a morphism $\phi: S^{2} \mathcal{E} \rightarrow \mathcal{O}_{X} \oplus \mathcal{E}$ is given by

$$
\begin{gathered}
\phi\left(z^{2}\right)=2\left(a^{2}-b d\right)+a z+b w \\
\phi(z w)=-(a d-b c)-d z-a w \\
\phi\left(w^{2}\right)=2\left(d^{2}-a c\right)+c z+d w
\end{gathered}
$$

where $S^{2} \mathcal{E}$ is the second symmetric power of $\mathcal{E}$ and $a, b, c, d \in \mathcal{O}_{X}$ with $b c \neq 0$. Here we remark that Miranda's construction also works for the case where $X$ and $Y$ are germs of analytic varieties even if $\mathcal{E}$ might fail to be a free $\mathcal{O}_{X}$-module.

Let $p: Y \rightarrow X$ be a covering map of degree 3 between two analytic varieties $X$ and $Y$ with $p_{*} \mathcal{O}_{Y} \cong \mathcal{O}_{X} \cdot\{1, z, w\}$. Then one can write

$$
\begin{aligned}
z^{2} & =g+a z+b w \\
z w & =h+e z+f w \\
w^{2} & =i+c z+d w
\end{aligned}
$$


for $a, b, \ldots, i \in \mathcal{O}_{X}$. Multiplying the equations in (3.2) by $w$ or $z$, and using the commutativity identities $z \cdot z w=w \cdot z^{2}$ and $z \cdot w^{2}=w \cdot z w$, we find

$$
g=b e+f^{2}-a f-b d, \quad h=b c-e f, \quad i=e^{2}+c f-a c-d e,
$$

(cf., [20, Lemma 2.4]). By the set up, no cubic polynomial in $z$ and $w$ has a square term, $z^{3}$ is generated by 1 and $z$ in $\mathcal{O}_{X}$ and, similarly, $w^{3}$ by 1 and $z$ in $\mathcal{O}_{X}$. So, (3.2) and (3.3) give

$$
\begin{aligned}
z^{3} & =a g+b h+\left(g+a^{2}+b e\right) z+(a b+b f) w, \\
w^{3} & =c h+d i+(c e+c d) z+\left(i+c f+d^{2}\right) w .
\end{aligned}
$$

Therefore, $a b+b f=0$ and $c e+c d=0$ on $\mathcal{O}_{X}$ yield $f=-a$ and $e=-d$ because, when $b=0$ (respectively, $c=0$ ) we have $z^{2}=g+a z$ (respectively, $w^{2}=i+d w$ ); this contradicts the fact that the field of fractions $K_{Y}$ over $\mathcal{O}_{Y}$ is an extension of $K_{X}$ of degree 3 (cf., [20, Lemma 2.6]).

Now, let us consider a triple cover $p: Y \rightarrow X$ where $X$ is smooth and $Y$ is defined by

$$
\begin{aligned}
& F(z, w):=z^{2}-2\left(a^{2}-b d\right)-a z-b w \\
& G(z, w):=z w+(a d-b c)+d z+a w \\
& H(z, w):=w^{2}-2\left(d^{2}-a c\right)-c z-d w
\end{aligned}
$$

with $a, b, c, d \in \mathcal{O}_{X}$.

Proposition 3.1. (see also [31]). With the preceding notation, the embedding of $Y$ into $\mathbb{C}^{2} \times X$ given by (3.4) is determinantal.

Proof. Recall that a (germ of an analytic) variety $V \subseteq \mathbb{C}^{N}$ is said to be determinantal if the ideal defining $V$ is generated by the $(t \times t)$-minors of an $(r \times s)$-matrix over $\mathcal{O}_{\mathbb{C}^{N}, 0}$ and $\operatorname{codim}(V)=$ $(r-t+1)(s-t+1)$ for $0<t \leq r \leq s$. The affirmation easily follows since the codimension of $Y$ in $\mathbb{C}^{2} \times X$ is 2 and the polynomials $F, G, H$ above can be written as the $(2 \times 2)$-minors of the matrix

$$
\left[\begin{array}{ccc}
z+a & w-2 d & c \\
b & z-2 a & w+d
\end{array}\right]
$$


It is easy to see that the variety defined by the $2 \times 2$-minors of $(3.5)$ is isomorphic to the one defined by the maximal minors of the matrix

$$
\left[\begin{array}{ccc}
z & w-3 d & c \\
b & z-3 a & w
\end{array}\right]
$$

under the transformation $(z, w) \mapsto(z+a, w+d)$. In what follows, we refer to either of them as Miranda's matrix form. Furthermore, we show that, when $X=\left(\mathbb{C}^{2}, 0\right), Y$ corresponds to an RTP-singularity for the appropriate choices of $a, b, c, d \in \mathcal{O}_{\mathbb{C}^{2}, 0}$.

4. Graphs of RTP-singularities. An RTP-singularity is a surface singularity which is rational with multiplicity 3 . The RTPsingularities are of nine types and are defined by three equations in $\mathbb{C}^{4}$. The explicit equations were first calculated by Tjurina in [28] using the minimal resolution graphs given by Artin in [2].

TABLE 1. Equations of the RTP-singularities.

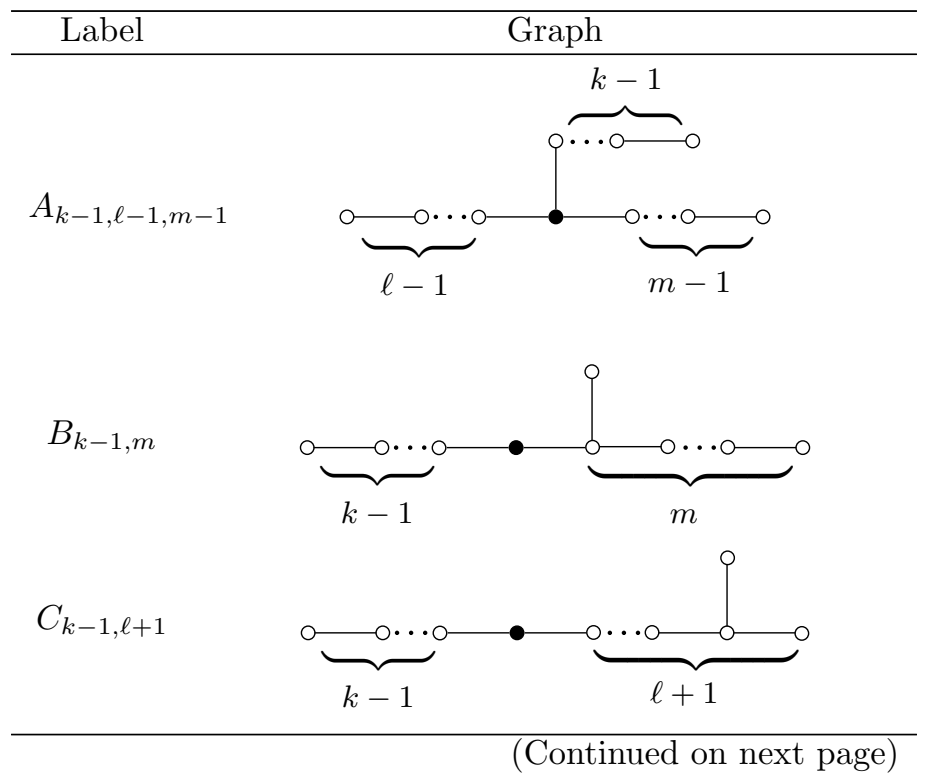


Table 1. (Continued from previous page)

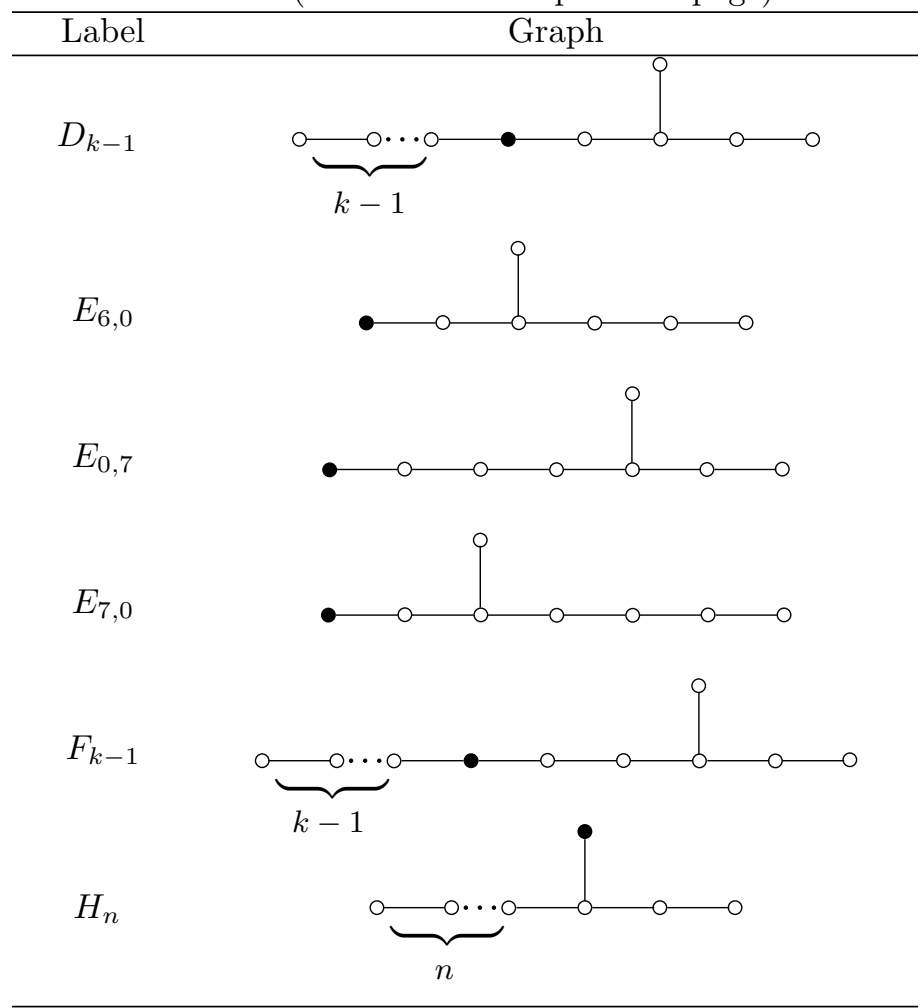

The classification of Artin is listed in Table 1 where the labels $E_{6,0}$, $E_{0,7}$ and $E_{7,0}$ are taken from [5] and the rest of them from Tjurina's work. The equations given by Tjurina provide beautiful examples to Miranda's construction of triple covers. Those equations can be obtained by taking $2 \times 2$-minors of the matrices listed in the second column of Table 2. The calculations required to transform the matrices into Miranda's form, which are shown in the third column of Table 2, are given in Appendix A. 
TABLE 2. Equations of the RTP-singularities.

\begin{tabular}{|c|c|c|}
\hline Label & $\begin{array}{c}\text { Matrices of } \\
\text { Tjurina's equations }\end{array}$ & Matrices in Miranda's form \\
\hline$A_{k-}$ & {$\left[\begin{array}{ccc}z & w & y^{m} \\
y^{k} & w+y^{\ell} & x\end{array}\right]$} & {$\left[\begin{array}{ccc}z & w-\left(x+y^{\ell}+y^{m}\right) & y^{m} \\
-y^{k} & z-x+y^{k} & w\end{array}\right.$} \\
\hline$B_{k-}$ & {$\left[\begin{array}{ccc}z & w+y^{\ell} & x y \\
y^{k} & x & w\end{array}\right]$} & {$\left[\begin{array}{ccc}z & w+y^{\ell} & x y \\
y^{k} & z+x & w-y^{k+1}\end{array}\right]$} \\
\hline$B_{k-}$ & {$\left[\begin{array}{ccc}z & w & x y+y^{\ell} \\
y^{k} & x & w\end{array}\right]$} & {$\left[\begin{array}{ccc}z & w & x y \\
y^{k} & z+x-y^{\ell-1} & w-y^{k+1}\end{array}\right]$} \\
\hline$C_{k-}$ & {$\left[\begin{array}{ccc}z & w & x^{\ell}+y^{2} \\
y^{k} & x & w\end{array}\right]$} & {$\left[\begin{array}{ccc}z & w+\ell x^{\ell-1} y^{k} & x^{\ell}+y^{2} \\
\alpha y^{k} & z+x & w\end{array}\right]$} \\
\hline$D$ & {$\left[\begin{array}{ccc}z & w+y^{2} & x^{2} \\
y^{k} & x & w\end{array}\right]$} & {$\left[\begin{array}{ccc}z & w+y^{2}+2 x y^{k} & x^{2} \\
y^{k} & z+x-y^{2 k} & w\end{array}\right]$} \\
\hline$E_{6,0}$ & {$\left[\begin{array}{ccc}z & w & x^{2} \\
y & z & w+y^{2}\end{array}\right]$} & {$\left[\begin{array}{ccc}z & w-y^{2} & x^{2} \\
y & z & w\end{array}\right]$} \\
\hline$E_{0,7}$ & {$\left[\begin{array}{ccc}z & w & x^{2}+y^{3} \\
y & z & w\end{array}\right]$} & {$\left[\begin{array}{ccc}z & w & x^{2}+y^{3} \\
y & z & w\end{array}\right]$} \\
\hline$E_{7,0}$ & {$\left[\begin{array}{ccc}z & w & y^{2} \\
y & z & w+x^{2}\end{array}\right]$} & {$\left[\begin{array}{ccc}z & w-x^{2} & y^{2} \\
y & z & w\end{array}\right]$} \\
\hline$F_{k-1}$ & {$\left[\begin{array}{ccc}z & w & x^{2}+y^{3} \\
y^{k} & x & w\end{array}\right]$} & {$\left[\begin{array}{ccc}z & w+2 x y^{k} & x^{2}+y^{3} \\
y^{k} & z+x-y^{2 k} & w\end{array}\right]$} \\
\hline$H_{3 k-1}$ & {$\left[\begin{array}{ccc}z & w & x y+y^{k} \\
x & z & w\end{array}\right]$} & {$\left[\begin{array}{ccc}z & w & x y+y^{k} \\
x & z & w\end{array}\right]$} \\
\hline$H_{3 k}$ & {$\left[\begin{array}{ccc}z & w & x y \\
x & z & w+y^{k}\end{array}\right]$} & {$\left[\begin{array}{ccc}z & w-y^{k} & x y \\
x & z & w\end{array}\right]$} \\
\hline$H_{3 k+1}$ & {$\left[\begin{array}{ccc}z & w+y^{k} & x \\
x y & z & w\end{array}\right]$} & {$\left[\begin{array}{ccc}z & w+y^{k} & x \\
x y & z & w\end{array}\right]$} \\
\hline
\end{tabular}

Remark 4.1. There is a direct way to calculate the minimal resolution graphs of RTP-singularities from Tjurina's equations due to Tjurina herself, namely, let $S$ be an RTP-singularity given by the maximal 
minors of the matrix

$$
\left[\begin{array}{lll}
f_{1} & f_{3} & f_{5} \\
f_{2} & f_{4} & f_{6}
\end{array}\right]
$$

and consider the embedding $S^{\prime} \subset \mathbb{C}^{4} \times \mathbb{P}^{1}$ defined by the equations

$$
t f_{1}=s f_{2}, \quad t f_{3}=s f_{4}, \quad t f_{5}=s f_{6},
$$

where $(s: t)$ are homogeneous coordinates in $\mathbb{P}^{1}([\mathbf{2 8}$, Section 2]). The surface $S^{\prime}$ is called the Tjurina modification of $S$ after [28]. It is locally a complete intersection singularity, and all of its singularities are rational. The map $\varphi:\left(S^{\prime}, E_{0}\right) \rightarrow(S, 0)$, which is induced by the natural projection $\mathbb{C}^{4} \times \mathbb{P}^{1} \rightarrow \mathbb{C}^{4}$, is birational and its fibre above the singular point 0 is the central curve $E_{0} \cong \mathbb{P}^{1}$ which corresponds to the exceptional curve with self intersection -3 in the minimal resolution graph. Moreover, the RDP-singularities connected to $E_{0}$ in the minimal resolution graph are the same type of singularities as the surface $\left(S^{\prime}, E_{0}\right)$ has at its singular points along $E_{0}$. Hence, one can deduce the minimal resolution graph of $(S, 0)$ by successive blow-ups of $\left(S^{\prime}, E_{0}\right)$.

4.1. Nonisolated forms of RTP-singularities. Now we aim to find hypersurface singularities in $\mathbb{C}^{3}$ such that their normalizations are the RTP-singularities. In this setting, normalization maps will be projections. By Theorem $[\mathbf{1 8}, 4.2 .1]$, there exists a Zariski dense open subset $U$ of the space of linear projections $\left(\mathbb{C}^{N}, 0\right) \rightarrow\left(\mathbb{C}^{3}, 0\right)$ such that, for every $p^{\prime} \in U$, the image $(X, 0)$ of $(S, 0)$ is a reduced hypersurface and the induced map $p:(S, 0) \rightarrow(X, 0)$ is finite and bimeromorphic. In order to form such a $p$, consider a projection $q:(S, 0) \rightarrow\left(\mathbb{C}^{2}, 0\right)$ which is finite and its kernel is transversal to the tangent cone of $S$ at 0 . The multiplicity of $S$ at 0 equals the smallest degree of $q$, i.e., the $\mathbb{C}$ vector space dimension of $\mathcal{O}_{S, 0} / q^{*} \mathfrak{m}_{\mathbb{C}^{2}, 0}$, by [22, Chapter 6, Corollary A.13]. Let $z: \mathbb{C}^{N} \rightarrow \mathbb{C}$ be a linear projection such that its restriction to $q^{-1}(y) \cap(S, 0)$ is injective for a regular value $y$ of $q$. Then, let $p:=(q, z)$.

When $(S, 0) \subset\left(\mathbb{C}^{4}, 0\right)$ is a surface defined by the ideal $I=(F, G, H)$, where $F, G$ and $H$ are as in (3.4) with $a, b, c, d \in \mathcal{O}_{\mathbb{C}^{2}, 0}$, the projection $q$ can be chosen to be the restriction of the Cartesian projection 
$(x, y, z, w) \mapsto(x, y)$ to $(S, 0)$. Furthermore, we can choose $p=(q, z)$ or $p=(q, w)$. Here we refer to a projection giving the nonisolated form of an RTP-singularity as suitable if the equation gives the expected minimal resolution graph by Oka's algorithm.

For example, the image of $p=(q, z)$ in $\mathbb{C}^{3}$ is the hypersurface

$$
(X, 0):=\left\{z^{3}+3\left(b d-a^{2}\right) z+\left(3 a b d-2 a^{3}-b^{2} c\right)=0\right\} .
$$

Moreover, $p$ is finite since $\operatorname{dim}_{\mathbb{C}} \mathcal{O}_{(S, 0)} / p^{*} \mathfrak{m}_{\mathbb{C}^{3}, 0}=2$ [9, Theorem 1.11].

On the other hand, the image of a linear projection of $S$ with an RTP-singularity is a surface $\left(X^{\prime}, 0\right)$ in $\mathbb{C}^{3}$ defined by an equation of the form

$$
z^{\nu}+f_{1}(x, y) z^{\nu-1}+\cdots+f_{\nu}(x, y)=0,
$$

for some positive integer $\nu<\infty$. The following proposition shows that we actually have $\nu=3$. A projection in $\mathbb{C}^{3}$ of a normal singularity is very useful for understanding deformations of the singularity (see, for example, $[6])$.

Proposition 4.2. A (suitable) projection of a surface defined by Tjurina's equation into $\mathbb{C}^{3}$ is one of the nonisolated hypersurfaces given by equations (i)-(ix) below.

(i) $A_{k-1, \ell-1, m-1}, k, \ell, m \geq 1$.

- $k \geq \ell \geq m$,

$$
z^{3}+x z^{2}-\left(x+y^{k}+y^{\ell}+y^{m}\right) y^{k} z+y^{2 k+\ell}=0,
$$

- $k=\ell<m$,

$$
z^{3}+\left(x-y^{k}\right) z^{2}-\left(x+y^{k}+y^{m}\right) y^{k} z+y^{2 k+m}=0 .
$$

(ii) $B_{k-1, m}, k \geq 2, m \geq 3$.

- $m=2 \ell$,

$$
z^{3}+x z^{2}-\left(y^{k+1}+y^{\ell}\right) y^{k} z-x y^{2 k+1}=0,
$$

- $m=2 \ell-1$,

$$
z^{3}+\left(x-y^{\ell-1}\right) z^{2}-y^{2 k+1} z-x y^{2 k+1}=0 .
$$


(iii) $C_{k-1, \ell+1}, k \geq 1, \ell \geq 2$,

$$
z^{3}+x z^{2}-\ell x^{\ell-1} y^{2 k} z-\left(x^{\ell}+y^{2}\right) y^{2 k}=0 .
$$

(iv) $D_{k-1}, k \geq 1$,

$$
z^{3}+\left(x+y^{2 k}\right) z^{2}+\left(2 x y^{k}-y^{2}\right) y^{k} z+x^{2} y^{2 k}=0 .
$$

(v) $E_{6,0}$,

$$
z^{3}+y^{3} z+x^{2} y^{2}=0
$$

(vi) $E_{0,7}$,

$$
z^{3}+y^{5}+x^{2} y^{2}=0
$$

(vii) $E_{7,0}$,

$$
z^{3}+x^{2} y z+y^{4}=0
$$

(viii) $F_{k-1}, k \geq 1$,

$$
z^{3}+\left(x+y^{2 k}\right) z^{2}+2 x y^{2 k} z+\left(x^{2}+y^{3}\right) y^{2 k}=0 .
$$

(ix) $H_{n}, n \geq 1$,

- $n=3 k-1$;

$$
z^{3}+x^{2} y\left(x+y^{k-1}\right)=0
$$

- $n=3 k$;

$$
z^{3}+x y^{k} z+x^{3} y=0
$$

- $n=3 k+1$;

$$
z^{3}+x y^{k+1} z+x^{3} y^{2}=0 .
$$

We will refer to these hypersurfaces in $\mathbb{C}^{3}$ as cubic surfaces.

Proof. Consider the equations obtained from the matrices in Miranda's form in Table 2 and the natural projection $(x, y, z, w) \mapsto$ $(x, y, z)$. Only the hypersurface equation for $A_{k-1, \ell-1, m-1}$, in the case $k \geq \ell \geq m$, requires an extra transformation of the form $x \mapsto x-y^{k}$ before the projection.

Remark 4.3. There are many projections one can apply to Tjurina's equations. However, not all of them have cubic surfaces as images. For 
example, the image of $F_{5}$ under the projection $(x, y, z, w) \mapsto(x, y, w)$ is an isolated singularity given by $\left\{-w^{2}+x y^{3}+x^{3}=0\right\}$. Note that the projection is not finite.

Remark 4.4. There also exist cubic surfaces in $\mathbb{C}^{3}$ whose normalizations are not rational. For instance, the image of the series

$$
\begin{aligned}
H_{k}:\left(\mathbb{C}^{2}, 0\right) & \longrightarrow\left(\mathbb{C}^{3}, 0\right) \\
(x, y) & \longmapsto\left(x, y^{3}, x y+y^{3 k-1}\right)
\end{aligned}
$$

is the variety $\left\{Z^{3}-3 X Y^{k} Z-X^{3} Y-Y^{3 k-1}=0\right\}$ (see Mond's classification in [21]). Its singular locus is also one-dimensional but the surface is not rational. Its normalization is, in fact, $\left(\mathbb{C}^{2}, 0\right)$.

4.2. Resolution of nonisolated forms by Newton polygons. In order to construct the minimal resolutions of RTP-singularities using Oka's theory [24], we start by recalling some notions needed. For details, see $[\mathbf{8}, \mathbf{1 0}]$.

Let $M$ be an integral lattice of rank $n$ with the standard basis $e_{1}, \ldots, e_{n}$ and $N$ its dual integral lattice. Let $M_{\mathbb{R}}:=M \otimes_{\mathbb{Z}} \mathbb{R}$ and $N_{\mathbb{R}}:=N \otimes_{\mathbb{Z}} \mathbb{R}$ be the corresponding real vector spaces. We will refer to the points of $N$ as integral vectors and a vector $\mathbf{u}=\left(u_{1}, \ldots, u_{n}\right) \in N_{\mathbb{R}}$ as primitive if all of its coordinates $u_{i}$ are coprime.

A nonempty subset $\sigma$ of $M_{\mathbb{R}}$ is called a cone if $\alpha \cdot \mathbf{u} \in \sigma$ exists for all $\mathbf{u} \in \sigma$ and $\alpha \in \mathbb{R}$. A convex polyhedral cone is the positive span of a finite set of vectors $\mathbf{u}_{1}, \ldots, \mathbf{u}_{k} \in M_{\mathbb{R}}$, that is,

$$
\sigma:=\left\{\sum_{i=1}^{k} \lambda_{i} \mathbf{u}_{i} \mid \mathbf{u}_{i} \in M_{\mathbb{R}}, \lambda_{i} \in \mathbb{R}_{\geq 0}\right\} .
$$

In this case, $\sigma$ is said to be generated by the vectors $\mathbf{u}_{1}, \ldots, \mathbf{u}_{k}$. A convex polyhedral cone generated only by integral vectors is rational and strongly convex if $\sigma \cap(-\sigma)=\{0\}$. The dimension of a cone $\sigma$ is the dimension of the linear space $\mathbb{R} \cdot \sigma$.

The dual of a convex polyhedral cone $\sigma$ is

$$
\check{\sigma}=\left\{\mathbf{v} \in N_{\mathbb{R}} \mid\langle\mathbf{u}, \mathbf{v}\rangle \geq 0, \text { for all } \mathbf{u} \in \sigma\right\} .
$$


A face $\tau$ of $\sigma$ is defined by

$$
\tau:=\{\mathbf{u} \in \sigma \mid\langle\mathbf{u}, \mathbf{v}\rangle=0, \mathbf{v} \in \check{\sigma} \cap N\} .
$$

In the rest of this section, $\sigma$ and the word "cone" will refer to a strongly convex rational polyhedral cone. We will also denote a cone with generators $\mathbf{u}_{1}, \ldots, \mathbf{u}_{k}$ by $\sigma_{\mathbf{u}_{1}, \ldots, \mathbf{u}_{k}}$ when we want to emphasise the generators.

Definition 4.5. The determinant of $\sigma_{\mathbf{u}_{1}, \ldots, \mathbf{u}_{k}}$ with $\mathbf{u}_{i}=\left(u_{1 i}, \ldots, u_{n i}\right)$, $i=1, \ldots, k$, is the greatest common divisor of $(k \times k)$-minors of the $(n \times k)$-matrix $U=\left(u_{i j}\right)$ and is $\operatorname{denoted}$ by $\operatorname{det}\left(\sigma_{\mathbf{u}_{1}, \ldots, \mathbf{u}_{k}}\right)$.

Definition 4.6. A cone $\sigma$ is regular if its determinant is equal to $\operatorname{det}(\sigma)= \pm 1$.

Definition 4.7. A fan $\mathcal{P}$ of dimension $n$ in $M_{\mathbb{R}}$ is a finite family of $n$-dimensional cones such that

(i) each face of a cone is also a cone in $\mathcal{P}$,

(ii) any intersection of two cones in $\mathcal{P}$ is a face of the two cones.

Example 4.8. Consider the three vectors $\mathbf{u}=(5,4,6), \mathbf{v}_{1}=(1,0,2)$ and $\mathbf{v}_{2}=(0,3,2)$ in $\mathbb{R}^{3}$. The fan $\mathcal{P}$ consisting of the cones $\sigma_{\mathbf{u e}_{1} \mathbf{v}_{1}}$, $\sigma_{\mathbf{u v}_{1} \mathbf{e}_{3} \mathbf{v}_{2}}$ and $\sigma_{\mathbf{u v}_{2} \mathbf{e}_{2} \mathbf{e}_{1}}$ is pictured in Figure 1 (A). Its section by the hyperplane $\{x+y+z=1\}$ is also drawn in Figure 1 (B).

Definition 4.9. A fan $\mathcal{P}$ is called regular if $\operatorname{det}(\sigma)= \pm 1$ for all $\sigma \in \mathcal{P}$.

Example 4.10. The fan illustrated in Figure 1 (A) is not regular as $\operatorname{det}\left(\sigma_{\mathbf{u e}_{1} \mathbf{v}}\right)=8$.

Remark 4.11. Suppose that $\mathbf{u}=(1,0, \ldots, 0)$ and $\mathbf{v}=\left(v_{1}, \ldots, v_{n}\right)$ are two primitive integral vectors generating $\sigma_{\mathbf{u v}}$. Then $s:=\operatorname{det}\left(\sigma_{\mathbf{u v}}\right)=$ $\operatorname{gcd}\left(v_{2}, \ldots, v_{n}\right)$ and $\operatorname{gcd}\left(s, v_{1}\right)=1$ as $\mathbf{v}$ is primitive. Let $\mathbf{t} \in \sigma_{\mathbf{u v}}$ with $\operatorname{det}\left(\sigma_{\mathbf{u t}}\right)=1$. Then there exist positive rational numbers $\alpha, \beta$ such that $\mathbf{t}=\alpha \mathbf{u}+\beta \mathbf{v}$. Observe that $\operatorname{det}\left(\sigma_{\mathbf{u t}}\right)=\operatorname{gcd}\left(\beta v_{2}, \ldots, \beta v_{n}\right)=\beta s$ 


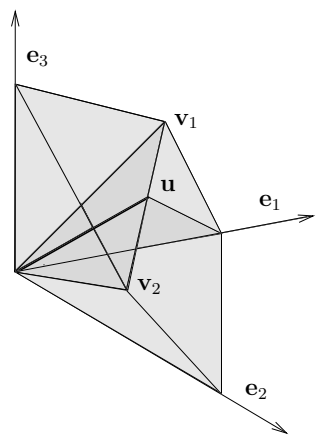

(A)

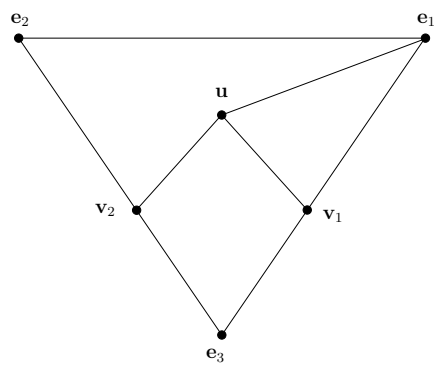

(B)

Figure 1. A fan formed by three dimensional cones in $\mathbb{R}^{3}$.

and $\operatorname{det}\left(\sigma_{\mathbf{t v}}\right)=\alpha s$ are integers. Thus the assumption $\operatorname{det}\left(\sigma_{\mathbf{u t}}\right)=1$ implies that $\mathbf{t}=(\mathbf{v}+\alpha s \cdot \mathbf{u}) / s$.

More generally, if a given fan is not regular it can be made regular by a process called regular subdivision.

4.2.1. Regular subdivision of a two-dimensional cone. Let $\sigma:=\sigma_{\mathbf{u v}}$ be a two-dimensional cone generated by two primitive vectors $\mathbf{u}, \mathbf{v}$ in $M_{\mathbb{R}}$. Assume that $s_{0}:=\operatorname{det}(\sigma)>1$. Then there exists a unique integral vector $(\mathbf{u v})_{1} \in \sigma$ between $\mathbf{u}$ and $\mathbf{v}$ defined by $(\mathbf{u v})_{1}=\left(\mathbf{v}+s_{1} \cdot \mathbf{u}\right) / s_{0}$ with

$$
s_{1}:=\operatorname{det}\left((\mathbf{u v})_{1}, \mathbf{v}\right) \in \mathbb{Z}_{\geq 0}, \quad 1 \leq s_{1}<s_{0}
$$

such that $\operatorname{det}\left(\mathbf{u},(\mathbf{u v})_{1}\right)=1$.

Proposition-Definition 4.12 ([24]). A regular subdivision of $\sigma$ is the finite decomposition

$$
\left\{(\mathbf{u v})_{0}=\mathbf{u},(\mathbf{u v})_{1}, \ldots,(\mathbf{u v})_{\alpha},(\mathbf{u v})_{\alpha+1}=\mathbf{v}\right\}
$$


where $(\mathbf{u v})_{i+1} \in \sigma_{(\mathbf{u v})_{i} \mathbf{v}}$ and is determined by the formula

$$
(\mathbf{u v})_{i+1}=\frac{\mathbf{v}+s_{i+1} \cdot(\mathbf{u v})_{i}}{s_{i}}
$$

and the conditions

$$
\begin{aligned}
s_{i+1} & :=\operatorname{det}\left((\mathbf{u v})_{i}, \mathbf{v}\right) \in \mathbb{Z}_{\geq 0}, \quad 1<s_{i+1}<s_{i} \\
& \text { for all } i=0, \ldots, \alpha-2 \text { and } s_{\alpha}=1 .
\end{aligned}
$$

Then we have $\operatorname{det}\left((\mathbf{u v})_{i},(\mathbf{u v})_{i+1}\right)=1$ for all $i=0, \ldots, \alpha$.

Example 4.13. Consider a two-dimensional cone $\sigma$ generated by the vectors $\mathbf{u}=(5,4,6)$ and $\mathbf{v}_{1}=(1,0,2)$ in $\mathbb{R}^{3}$ (see Example 4.8). We have $s_{0}=\operatorname{det}(\sigma)=\operatorname{gcd}(8,4,4)=4>1$. We find

$$
\begin{aligned}
& \left(\mathbf{u v}_{1}\right)_{1}=\frac{\mathbf{v}_{1}+s_{1} \cdot \mathbf{u}}{s_{0}}=(4,3,5), \quad s_{1}=3, \\
& \left(\mathbf{u v}_{1}\right)_{2}=\frac{\mathbf{v}_{1}+s_{2} \cdot\left(\mathbf{u v}_{1}\right)_{1}}{s_{1}}=(3,2,4), \quad s_{2}=2, \\
& \left.\mathbf{u v}_{1}\right)_{3}=\frac{\mathbf{v}_{1}+s_{3} \cdot(\mathbf{u v})_{2}}{s_{2}}=(2,1,3), \quad s_{3}=1 .
\end{aligned}
$$

Hence, the decomposition $\left\{\mathbf{u},\left(\mathbf{u v}_{1}\right)_{1},\left(\mathbf{u v}_{1}\right)_{2},\left(\mathbf{u v}_{1}\right)_{3}, \mathbf{v}_{1}\right\}$ is the regular subdivision of $\sigma$.

4.2.2. Algorithm for constructing a graph from a threedimensional fan. Following Oka's theory in [24] (see also subsection 4.2.3), one can associate a graph to any regular subdivision of a fan as follows.

Let $\mathcal{P}$ be a fan in $M_{\mathbb{R}} \cap \mathbb{R}_{\geq 0}^{3}$ with generators $\mathbf{u}_{1}, \ldots, \mathbf{u}_{k} \in \mathbb{N}^{\oplus 3}$ such that each $\mathbf{u}_{i}$ is a face of a two-dimensional cone in $\mathcal{P}$. Then

Step 1a. Pick a generator $\mathbf{u}:=\mathbf{u}_{i} \in \mathcal{P} \cap(\mathbb{N}-\{0\})^{\oplus 3}$. Consider all two-dimensional cones $\sigma_{\mathbf{u v}_{1}}, \ldots, \sigma_{\mathbf{u v}_{l}} \in \mathcal{P}$ which are adjacent to $\sigma_{\mathbf{u}}$ in $\mathcal{P}$, i.e. $\sigma_{\mathbf{u v}_{i}} \cap \sigma_{\mathbf{u v}_{j}}=\sigma_{\mathbf{u}}, i \neq j$, for $\sigma_{\mathbf{v}_{j}} \in \mathcal{P}, j=1, \ldots l$.

Step 1b. For each two-dimensional cone $\sigma_{\mathbf{u v}_{j}} \in \mathcal{P}, j=1, \ldots, l$, find the regular subdivision $\left\{\left(\mathbf{u v}_{j}\right)_{0}=\mathbf{u}, \ldots,\left(\mathbf{u v}_{j}\right)_{\alpha_{i}},\left(\mathbf{u v}_{j}\right)_{\alpha_{i}+1}=\right.$ $\left.\mathbf{v}_{j}\right\}$. 
Step 1c. Construct a tree $\Gamma_{\mathbf{u}}^{j}$ as follows. Assign the vertices $V_{0}^{(j)}$, $V_{1}^{(j)}, \ldots, V_{\alpha_{i}+1}^{(j)}$ to the vectors $\left(\mathbf{u v}_{j}\right)_{0}, \ldots,\left(\mathbf{u v}_{j}\right)_{\alpha_{i}+1}$, respectively, and draw an edge between $V_{i}^{(j)}$ and $V_{i+1}^{(j)}$ for all $i=0, \ldots, \alpha_{i}$. Note that $V_{0}^{(j)}=V_{0}^{(1)}$ since $\left(\mathbf{u v}_{j}\right)_{0}=\mathbf{u}$ for all $j$. Let $V_{0}:=V_{0}^{(1)}$.

Step 1d. Erase the vertex $V_{\alpha_{j}+1}^{(j)}$ and its adjacent edges such that the graph $\Gamma_{\mathbf{u}}^{j}$ remains connected if its associated vector $\mathbf{v}_{j}$ is not strictly positive.

Step 2. Glue all $\Gamma_{\mathbf{u}}^{j}$ along the common vertex $V_{0}$ to obtain an abstract graph $\Gamma_{\mathbf{u}}$ corresponding to $\mathbf{u}$.

Step 3. Find the graph $\Gamma_{\mathbf{u}_{i}}$ for each strictly positive generator $\mathbf{u}_{i}$ of $\mathcal{P}$ and glue $\Gamma_{\mathbf{u}_{i}}$ to $\Gamma_{\mathbf{u}_{j}}$ for each $i \neq j$ along their common vertices, if they exist. The resulting connected graph is the graph of the fan $\mathcal{P}$, denoted by $\Gamma_{\mathcal{P}}$, cf., [24].

Example 4.14. Let $\mathcal{P}$ be the fan studied in Example 4.8. The regular subdivision of $\sigma_{\mathbf{u v}_{1}}$ is given by

$$
\left\{\mathbf{u},\left(\mathbf{u v}_{1}\right)_{1},\left(\mathbf{u v}_{1}\right)_{2},\left(\mathbf{u v}_{1}\right)_{3}, \mathbf{v}_{1}\right\}=\left\{\mathbf{u},(4,3,5),(3,2,4),(2,1,3), \mathbf{v}_{1}\right\},
$$

see Example 4.13. The corresponding graph $\Gamma_{\mathbf{u}}^{1}$ is then a tree consisting of five vertices as shown in Figure $2(\mathrm{~A})$.

The regular subdivision of $\sigma_{\mathbf{u v}_{2}}$ is

$$
\left\{\mathbf{u},\left(\mathbf{u v}_{2}\right)_{1},\left(\mathbf{u v}_{2}\right)_{2}, \mathbf{v}_{2}\right\}=\left\{\mathbf{u},(3,3,4),(1,2,2), \mathbf{v}_{2}\right\} .
$$

Then $\Gamma_{\mathbf{u}}^{2}$ is a tree with four vertices (see Figure $2(\mathrm{~B})$ ). Finally, the regular subdivision of $\sigma_{\mathbf{u e}_{1}}$ is $\left\{\mathbf{u},\left(\mathbf{u e}_{1}\right)_{1}, \mathbf{e}_{1}\right\}=\left\{\mathbf{u},(3,2,3), \mathbf{e}_{1}\right\}$. So, we get the graph $\Gamma_{\mathbf{u}}^{3}$ with three vertices as shown in Figure $2(\mathrm{C})$. Therefore, to construct the graph for $\mathcal{P}$, we delete the vertices $V_{4}^{(1)}$, $V_{3}^{(2)}$ and $V_{2}^{(3)}$ which correspond to non-strictly positive vectors $\mathbf{v}_{1}$, $\mathbf{v}_{2}$ and $\mathbf{e}_{1}$, respectively. Then we glue $\Gamma_{\mathbf{u}}^{1}, \Gamma_{\mathbf{u}}^{2}$ and $\Gamma_{\mathbf{u}}^{3}$ by overlapping the vertices $V_{0}^{(1)}=V_{0}^{(2)}=V_{0}^{(3)}$. This gives the graph $\Gamma_{\mathcal{P}}$ shown in Figure 3.

Remark 4.15. One can assign a weight and a genus to each vertex of any abstract connected graph in a way that the intersection matrix associated with the graph is negative definite. We can obtain a 


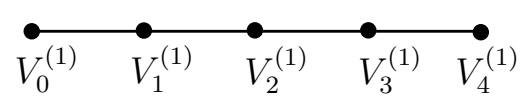

(A) $\Gamma_{\mathbf{u}}^{1}$

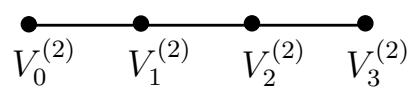

(B) $\Gamma_{\mathbf{u}}^{2}$

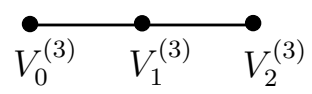

(C) $\Gamma_{\mathbf{u}}^{3}$

Figure 2. Examples of trees.

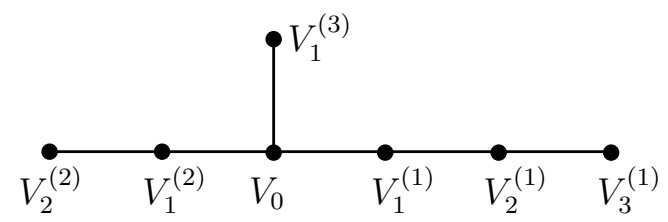

FiguRE 3. Graph corresponding to the fan in Example 4.8.

configuration of curves by associating a curve to each vertex in the graph and intersecting any two corresponding curves if there exists an edge between them. By plumbing construction around such a configuration, we can embed the configuration into an analytic surface $\tilde{X}$. As it has the negative definite intersection matrix, $\widetilde{X}$ becomes a resolution of an analytic surface singularity [11]. For example, the graph given in Figure 3 with weight 2 and genus 0 assigned to each vertex is the minimal resolution graph of RDP-singularity of type $E_{7}$. Note that the graph in Figure 3 will also represent the minimal resolution graph of the RTP-singularity of type $E_{6,0}$, see Table 1. Recall also that RDP and RTP-singularities are taut, that is, the surface obtained by plumbing construction for any of the resolution graphs of these types is unique up to an analytic isomorphism $[\mathbf{2}, \mathbf{1 7}]$.

By using combinatorial data (as in Remark 2.5), we can relate such a construction to a hypersurface singularity if we choose the fan $\mathcal{P}$ to be the dual Newton polygon of the equation defining the singularity. 
4.2.3. Newton polygon of a singularity. Let

$$
f(\mathbf{z}):=\sum_{\mathbf{v}} a_{\mathbf{v}} \mathbf{z}^{\mathbf{v}} \in \mathcal{O}_{\mathbb{C}^{n}, 0}
$$

where $\mathbf{z}^{\mathbf{v}}=z_{1}^{v_{1}} \cdots z_{n}^{v_{n}}$ with $\mathbf{v}=\left(v_{1}, \ldots, v_{n}\right) \in \mathbb{N}^{n}$. The support of $f$ is the set

$$
\operatorname{supp} f:=\left\{\mathbf{v} \in \mathbb{N}^{n} \mid a_{\mathbf{v}} \neq 0\right\} .
$$

Definition 4.16. The Newton polygon $N P(f)$ of $f$ is the boundary of the convex closure in $M_{\mathbb{R}}$ of

$$
\bigcup_{\mathbf{v} \in \operatorname{supp} f}\left\{\mathbf{v}+\mathbb{R}_{\geq 0}^{n}\right\} \subseteq M_{\mathbb{R}}
$$

Definition 4.17. Given a vector $\mathbf{u} \in N_{\mathbb{R}} \cap \mathbb{R}_{\geq 0}^{n}$, the face of $N P(f)$ with respect to $\mathbf{u}$ is defined as

$$
F_{\mathbf{u}}:=\left\{\mathbf{v} \in N P(f) \mid\langle\mathbf{u}, \mathbf{v}\rangle=\min _{\mathbf{w} \in N P(f)}\langle\mathbf{u}, \mathbf{w}\rangle\right\} \subseteq M_{\mathbb{R}} .
$$

Note that $\mathbf{u}$ is normal to the face $F_{\mathbf{u}}$. Let us refer to the $(n-1)$ dimensional faces of a polygon as facets. Let us define an equivalence relation on $N_{\mathbb{R}} \cap \mathbb{R}_{\geq 0}^{n}$ by

$$
\mathbf{u} \sim \mathbf{u}^{\prime} \quad \text { if and only if } \quad F_{\mathbf{u}}=F_{\mathbf{u}^{\prime}} .
$$

Then each equivalence class forms a cone structure in $N_{\mathbb{R}} \cap \mathbb{R}_{\geq 0}^{n}$. In fact, these cones form a fan; it is called the dual fan of $N P(\bar{f})$ and denoted by $D N P(f)$. Hence, there is a one-to-one correspondence between the cones of $D N P(f)$ and the faces of $N P(f)$.

Definition $4.18([24])$. An analytic function

$$
f(\mathbf{z})=\sum_{\mathbf{v}} a_{\mathbf{v}} \mathbf{z}^{\mathbf{v}} \quad \text { in } \mathcal{O}_{\mathbb{C}^{n}, 0}
$$

is non-degenerate with respect to its Newton polyhedron in coordinates $\left(z_{1}, \ldots, z_{n}\right)$ or in short Newton non-degenerate if, for all compact faces $F_{\mathbf{u}}$ associated to a non-zero vector $\mathbf{u}$ of $N P(f)$, the face function or 
the initial form

$$
\operatorname{In}_{\mathbf{u}}(f):=\sum_{\mathbf{v} \in F_{\mathbf{u}}} a_{\mathbf{v}} \mathbf{z}^{\mathbf{v}}
$$

defines a nonsingular hypersurface in the torus $\left(\mathbb{C}^{*}\right)^{n}$.

Example 4.19. Consider the RTP-singularity $F_{k-1}$ defined by the equation $f$ given by Proposition $4.2(\mathrm{v})$. The $N P(f)$ has two compact faces $F_{\mathbf{u}_{1}}$ and $F_{\mathbf{u}_{2}}$, see Table 3 . Then the face functions of $f$ are

$$
\operatorname{In}_{\mathbf{u}_{1}}=z^{3}+x z^{2}+y^{2 k+3}, \quad \operatorname{In}_{\mathbf{u}_{2}}=x z^{2}+x^{2} y^{2 k}+y^{2 k+3} .
$$

The Jacobian ideals are

$$
\begin{aligned}
& J\left(\operatorname{In}_{\mathbf{u}_{1}}\right)=\left(2 x z,(2 k+3) y^{2 k+2}, 3 z^{2}+2 x z\right), \\
& J\left(\operatorname{In}_{\mathbf{u}_{2}}\right)=\left(z^{2}+2 x y^{2 k},(2 k+3) y^{2 k+2}+(2 k) x^{2} y^{2 k-1}, 2 x z\right) .
\end{aligned}
$$

Clearly, none of the varieties which are defined by $\operatorname{In}_{\mathbf{u}_{1}}$ and $\operatorname{In}_{\mathbf{u}_{2}}$ has a singularity in $\left(\mathbb{C}^{*}\right)^{3}$. Hence, $F_{k-1}$ is non-degenerate.

When $f$ is non-degenerate, then a toric modification associated to a regular subdivision of $N P(f)$ resolves the singularity defined by $f$. In the case of isolated surface singularities, Oka's results provide a canonical way to obtain a resolution graph. Before stating his construction, we need the following definition.

Definition 4.20. Define an integer $g(\mathbf{u})$ to be the number of integer points in the interior of the face $F_{\mathbf{u}}$ and $r\left(\sigma_{\mathbf{u v}}\right)$ to be the number of integer points in the interior of the face $F_{\mathbf{u}} \cap F_{\mathbf{v}}$ in $N P(f)$.

Definition 4.21 (Oka's algorithm, $[24]$ ). Let $X$ be a two-dimensional hypersurface defined by $f \in \mathcal{O}_{\mathbb{C}^{3}, 0}$ with an isolated singularity at the origin. Oka's algorithm for constructing the resolution graph $\Gamma_{f}$ of a resolution of $X$ consists of two steps. First, construct $\Gamma_{f}$ by applying the algorithm in subsection 4.2.2 to $D N P(f)$ with an additional operation: glue $r\left(\sigma_{\mathbf{u v}_{j}}\right)+1$ copies of the trees $\Gamma_{\mathbf{u}}^{j}$ along their beginning vertices. Second, associate weights $w_{1}, \ldots, w_{\alpha}$ to each 
$\Gamma_{\mathbf{u}}^{j}$ defined by the continuous fraction

$$
\left[w_{1}: \cdots: w_{\alpha}\right]:=\frac{s}{s_{1}}=w_{1}-\frac{1}{w_{2}-\frac{1}{\ddots \cdot-\frac{1}{w_{\alpha}}}},
$$

where $s=\operatorname{det}\left(\sigma_{\mathbf{u v}_{j}}\right)$ and $s_{1}$ is defined as in subsection 4.2.1. A weight $w_{\mathbf{u}}$ of $\mathbf{u}$ is defined by the equation

$$
w_{\mathbf{u}} \cdot \mathbf{u}=\sum_{j=1}^{l}\left(r\left(\sigma_{\mathbf{u v}_{j}}\right)+1\right) \cdot\left(\mathbf{u v}_{j}\right)_{1},
$$

where $l$ is the number of two-dimensional cones $\sigma_{\mathbf{u v}_{j}}$ for which $\mathbf{u}$ is one of the generators.

Then, the vertices corresponding to $\mathbf{u}$ represent the components of $E$ with genus $g(\mathbf{u})$ and self intersection numbers $-w_{\mathbf{u}}$, and all the others, i.e., the vertices in $\Gamma_{f}$ coming from the vectors added in the process of regular subdivision represent the components of $E$ with genus 0 and self intersection numbers $-w_{i}$ calculated by formula (4.2).

Note that the value of $r\left(\sigma_{\mathbf{u v}_{j}}\right)$ can be obtained in a different way as follows.

Definition 4.22. With the notation in subsection 4.2 .2 , let $\mathbf{u} \in \mathcal{P}$ be a strictly positive vector. Let us take the projection of $\mathcal{P}$ onto the plane, $\mathbf{u}$ normal, to get the vectors $\widetilde{\mathbf{v}}_{1}, \ldots, \widetilde{\mathbf{v}}_{l}$, which are not necessarily strictly positive. We define the constants $c_{1}, \ldots, c_{l} \in \mathbb{N}-\{0\}$ to be the minimal solution of $\sum c_{j} \widetilde{\mathbf{v}}_{j}=0$.

By direct calculation, we have

Lemma 4.23. For an RTP-singularity, we have $c_{j}=r\left(\sigma_{\mathbf{u v}_{j}}\right)+1$ for all $j$.

Example 4.24. Consider the surface singularity of type $E_{6,0}$ defined in $\mathbb{C}^{3}$ by $f(x, y, z):=z^{3}+y^{3} z+x^{2} y^{2} \in \mathcal{O}_{\mathbb{C}^{3}, 0}$. The support of $f$ is 
the set $\operatorname{supp} f=\{(2,2,0),(0,3,1),(0,0,3)\}$ and the Newton polygon $N P(f) \subseteq \mathbb{R}^{3}$ which is shown in Figure 4. We see that $N P(f)$ has one compact facet $F_{\mathbf{u}}$ and five non-compact facets $F_{\mathbf{e}_{1}}, F_{\mathbf{e}_{2}}, F_{\mathbf{e}_{3}}, F_{\mathbf{v}_{1}}, F_{\mathbf{v}_{2}}$ with the normal vectors $\mathbf{u}=(5,4,6), \mathbf{e}_{1}=(1,0,0), \mathbf{e}_{2}=(0,1,0)$, $\mathbf{e}_{3}=(0,0,1)$ and $\mathbf{v}_{1}=(1,0,2), \mathbf{v}_{2}=(0,3,2)$, respectively. Hence, the dual space $D N P(f)$ is the fan given in Figure 1 which was studied in Examples 4.14.

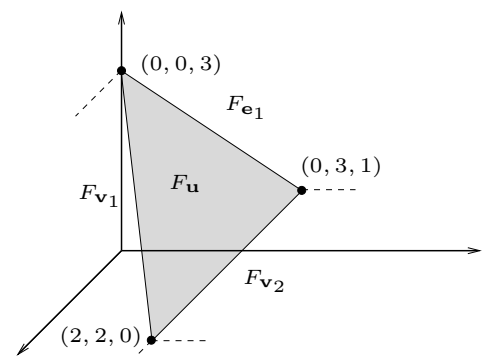

FIGURE 4. Newton polygon of the nonisolated form of $E_{6,0}$.

The weights for the subdivision of $\sigma_{\mathbf{u v}_{1}}$ are [2:2:2]; for $\sigma_{\mathbf{u v}_{2}}$, they are [2:3] and for $\sigma_{\mathbf{u e}_{1}}$, the weight is [2]. The central vertex corresponding to $\mathbf{u}$ has weight 2 . Furthermore, $g(\mathbf{u})=0$ and $r(\sigma)=0$ for all two-dimensional cones in $D N P(f)$. Hence, Oka's algorithm applied to the nonisolated form of $E_{6,0}$ yields the graph in Figure 3 which is the minimal resolution graph of $E_{6,0}$ by Artin's classification (see also Table 1).

Remark 4.25. If a fan is not a dual Newton polygon of a function, the weight formula (4.3) or Definition 4.22 may not always yield integers. For instance, consider the fan $\mathcal{P}^{\prime} \subset \mathbb{R}^{3}$ pictured in Figure 5. Each two-dimensional cone in $\mathcal{P}^{\prime}$ is regular. The weight corresponding to the vector $(1,1,1)$ is 3 , the others are 2 . So, the related graph $\Gamma_{\mathcal{P}^{\prime}}$ consists of five vertices. We observe that there is no solution to the weight formula

$$
w_{(3,2,3)} \cdot(3,2,3)=\alpha_{1} \cdot(1,1,1)+\alpha_{2} \cdot(2,1,2)+\alpha_{3} \cdot(2,2,3)
$$

with $w_{(3,2,3)}, \alpha_{1}, \alpha_{2}, \alpha_{3} \in \mathbb{N}-\{0\}$. Instead, we can deduce a suitable 


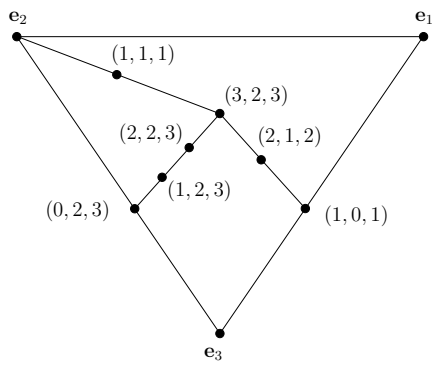

FiguRe 5. A fan $\mathcal{P}$.

weight by the following fact for graphs

$$
\begin{aligned}
& -a_{j} w_{j}+\sum_{i} a_{i} \geq 0, \quad \text { for all } j, \\
& -a_{j} w_{j}+\sum_{i} a_{i}>0, \quad \text { for some } j,
\end{aligned}
$$

where the sum is taken over all $i$ such that the $i$ th vertex is connected to the $j$ th vertex by an edge. The existence of such $a_{i}$ 's is due to Laufer's algorithm [16] for the computation of Artin's divisor. For example, we can choose $w_{(3,2,3)} \geq 3$ with $a=1$ or $w=2$ with $a=2$ to relate the graph with a rational singularity (see also [19]).

Recall that we have chosen a suitable projection to obtain the nonisolated forms in order to find the minimal resolution graphs for each singularity given by [2]. We observed by some elementary but time consuming calculations that the nonisolated forms of RTP-singularities given in Proposition 4.2 are all non-degenerate singularities. Moreover,

Proposition 4.26. The minimal resolution graphs of nonisolated forms of RTP-singularities defined by the equations (i)-(ix) in Proposition 4.2 can be obtained by Oka's algorithm followed by a number of blow-downs and coincide with Artin's classification in [2]. 
Proof. Simply, apply Oka's algorithm to the equations. See Table 3 for the main steps. Note that $g(\mathbf{u})=0$ for all the series. The integers $r(\sigma)$ are all equal to 0 except in the following cases. For the series $A_{k-1, \ell-1, m-1}, r\left(\sigma_{\mathbf{u}_{1} \mathbf{e}_{1}}\right)=1$ if $k=\ell<m$ or if $k \geq \ell \geq m$ and $k, m$ are both even or odd but $k \neq m, r\left(\sigma_{\mathbf{u}_{1} \mathbf{e}_{1}}\right)=2$ if $k=\ell=m$; for $B_{k-1,2 \ell}$, $r\left(\sigma_{\mathbf{u}_{1} \mathbf{e}_{1}}\right)=1$; for $C_{k-1, \ell+1}, r\left(\sigma_{\mathbf{u}_{1} \mathbf{e}_{1}}\right)=2$ if $k=3 p+2, r\left(\sigma_{\mathbf{u}_{2} \mathbf{e}_{3}}\right)=1$ if $\ell$ is even and $r\left(\sigma_{\mathbf{u}_{2} \mathbf{v}}\right)=1$ if $\ell$ is odd; for $D_{k-1}, r\left(\sigma_{\mathbf{u}_{1} \mathbf{e}_{1}}\right)=1$ if $k$ is even; finally, for $F_{k-1}, r\left(\sigma_{\mathbf{u}_{1} \mathbf{e}_{1}}\right)=2$ if $k=3 p$.

Moreover, the weight of $\mathbf{u}_{1}$ is equal to 1 in the following cases: $A_{k-1, \ell-1, m-1}$ if $k \geq \ell \geq m$ and $k$ is odd, $m$ is even or $k$ is even, $m$ is odd; $B_{k-1,2 \ell}$ if $k$ is odd, $\ell$ is even or $k$ is even, $\ell$ is odd; $D_{k-1}$ if $k$ is odd and $k>1 ; F_{k-1}$ if $k=3 p+1$ and $k>1$ or $k=3 p+2$. Therefore, those require one blow-down after the process. We also find $w_{\mathbf{u}_{1}}=1$ for the series $C_{k-1, \ell+1}$ when $k=3 p$ or $k=3 p+1$. However, one needs to apply two successive blow-downs. On the other hand, $w_{\mathbf{u}_{3}}=1$ for $B_{k-1,2 \ell-1}$ in which case one needs $k-\ell+1$ successive blow-downs to find the minimal resolution graph. This concludes the proof.

Let $S$ be an RTP-singularity defined by the maximal minors of one of the matrices given in Table 2 and $X$ its nonisolated form given in Proposition 4.2. Let $\widetilde{X}$ be the resolution of $X$ by Oka's process. Then, by contracting the $(-1)$-curves on $\widetilde{X}$ using Castelnuovo criterion we get the minimal resolution $\widetilde{S}$ of $S$. Therefore, we have the commutative diagram

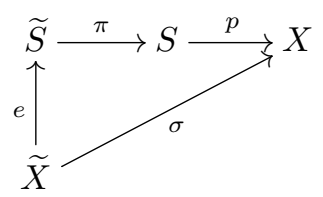

where $\pi$ is the minimal resolution, $p$ is the projection which is also a normalization, $e$ is the contraction map and $\sigma$ is the resolution of $X$ obtained by Oka's process.

Remark 4.27. As one can expect, many cubic equations may give the same Newton polygon. However, they may not come from a projection 
of an RTP-singularity. For example, the hypersurface

$$
z^{3}+x z^{2}-y^{2 k+1} z-x y^{2 k+1}=0
$$

has the same Newton polygon as $B_{k-1,2 k+2}$, but its normalization is smooth. So, it is not a nonisolated form of an RTP-singularity listed in Proposition 4.2. Moreover, it is degenerate. Its Newton polygon has one compact face. Hence, the degeneracy of (4.5) follows from the fact that it has singularities in the torus.

The concept of non-degeneracy in a more general setting is defined as follows.

Definition $4.28([\mathbf{1}])$. For an ideal $I$ in $\mathcal{O}_{\mathbb{C}^{n}, 0}$, define the initial ideal by $\operatorname{In}_{\mathbf{u}}(I)=\left\langle\operatorname{In}_{\mathbf{u}}(f) \mid f \in I\right\rangle$. Then, an affine variety $V(I) \subseteq \mathbb{C}^{n}$ is said to be Newton non-degenerate if for every $\mathbf{u} \in \mathbb{R}_{\geq 0}^{n}, V\left(\operatorname{In}_{\mathbf{u}} I\right)$ does not have any singularity in $\left(\mathbb{C}^{*}\right)^{n}$.

Remark 4.29. Non-degeneracy of a hypersurface singularity in $\mathbb{C}^{3}$ can be checked by simple calculations as in Example 4.19. However, it might be very useful to work with computer programs such as GFAN [14] and Singular [7] when one studies ideals in higher dimensions.

Remark 4.30. An explicit computation shows that all of the nonisolated forms of RTP-singularities listed in Proposition 4.2 are Newton non-degenerate.

In fact, we have

Theorem 4.31. The nonisolated forms of RTP-singularities and their normalizations are Newton non-degenerate.

Hence, we are inspired to suggest the following conjecture.

Conjecture 4.32. A normal surface singularity is Newton nondegenerate if and only if it is a normalization of a nonisolated nondegenerate hypersurface singularity. 
In a forthcoming paper, we will show that a rational singularity of multiplicity $m \geq 4$ can be written as (4.1) with $\nu=m$ by some projection. Note that not all rational singularities are determinantal. For example, there are minimal resolution graphs in the classification of rational singularities which contain a vertex with weight $\geq 3$ and coefficient $\geq 2$ in Artin's divisor of the resolution.

TABLE 3. Resolution process of the RTP-singularities.

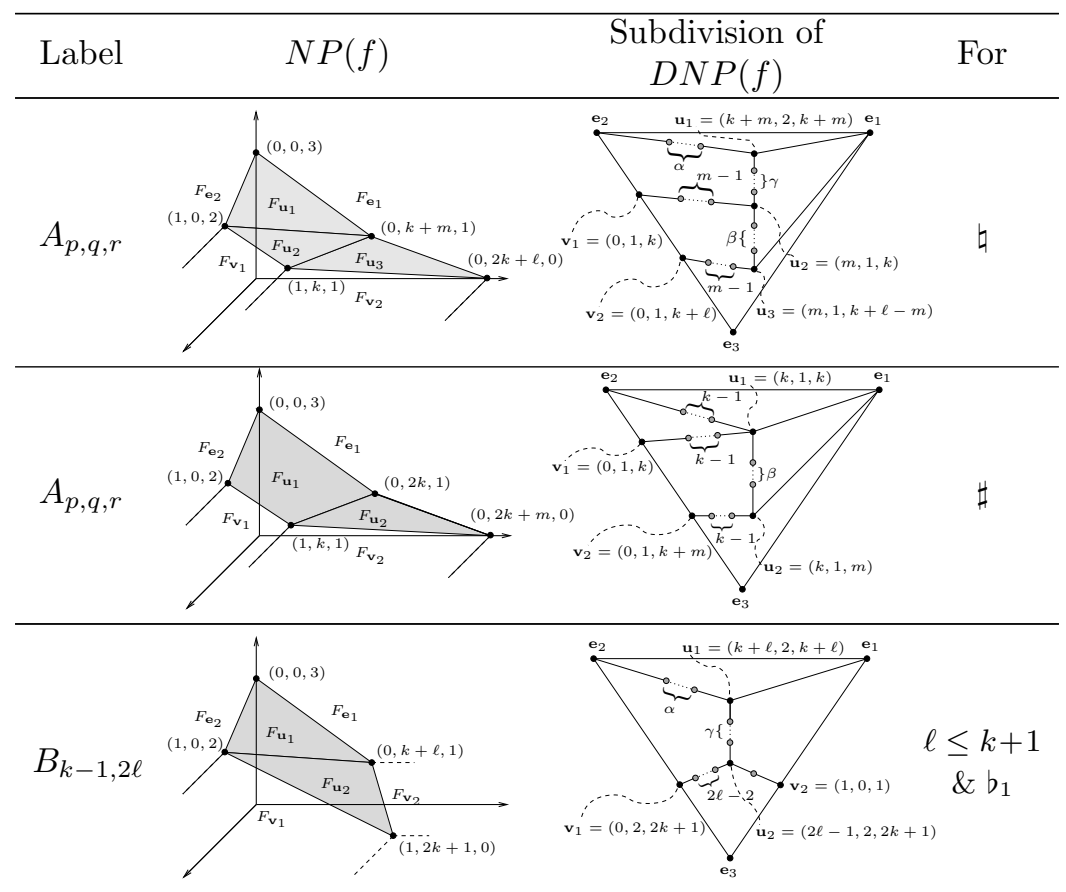

घ: $p=k-1, q=\ell-1, r=m-1, k \geq \ell \geq m, \beta=\ell-m-1 ; \alpha=\frac{k+m-2}{2}$, $\gamma=\frac{k-m-2}{2}$ if $k, m$ both even or odd but $k \neq m ; \alpha=\frac{k+m-1}{2}, \gamma=\frac{k-m-1}{2}$ if $k$ odd, $m$ even or $k$ even $m$ odd; $\mathbf{u}_{1}=\mathbf{u}_{2}=\mathbf{u}_{3}$ and $\alpha=k-1, \gamma=\beta=0$ if $k=\ell=m$.

$\sharp: p=k-1, q=\ell-1, r=m-1, k=\ell<m, \beta=k-m-1$.

$b_{1}: \alpha=\frac{k+\ell-2}{2}, \gamma=\frac{k-\ell}{2}$ if $k, \ell$ both even or odd; $\alpha=\frac{k+\ell-1}{2}$ and $\gamma=\frac{k-\ell+1}{2}$ if $k$ odd, $\ell$ even or $k$ even $\ell$ odd. 
Table 3. (Continued from previous page)

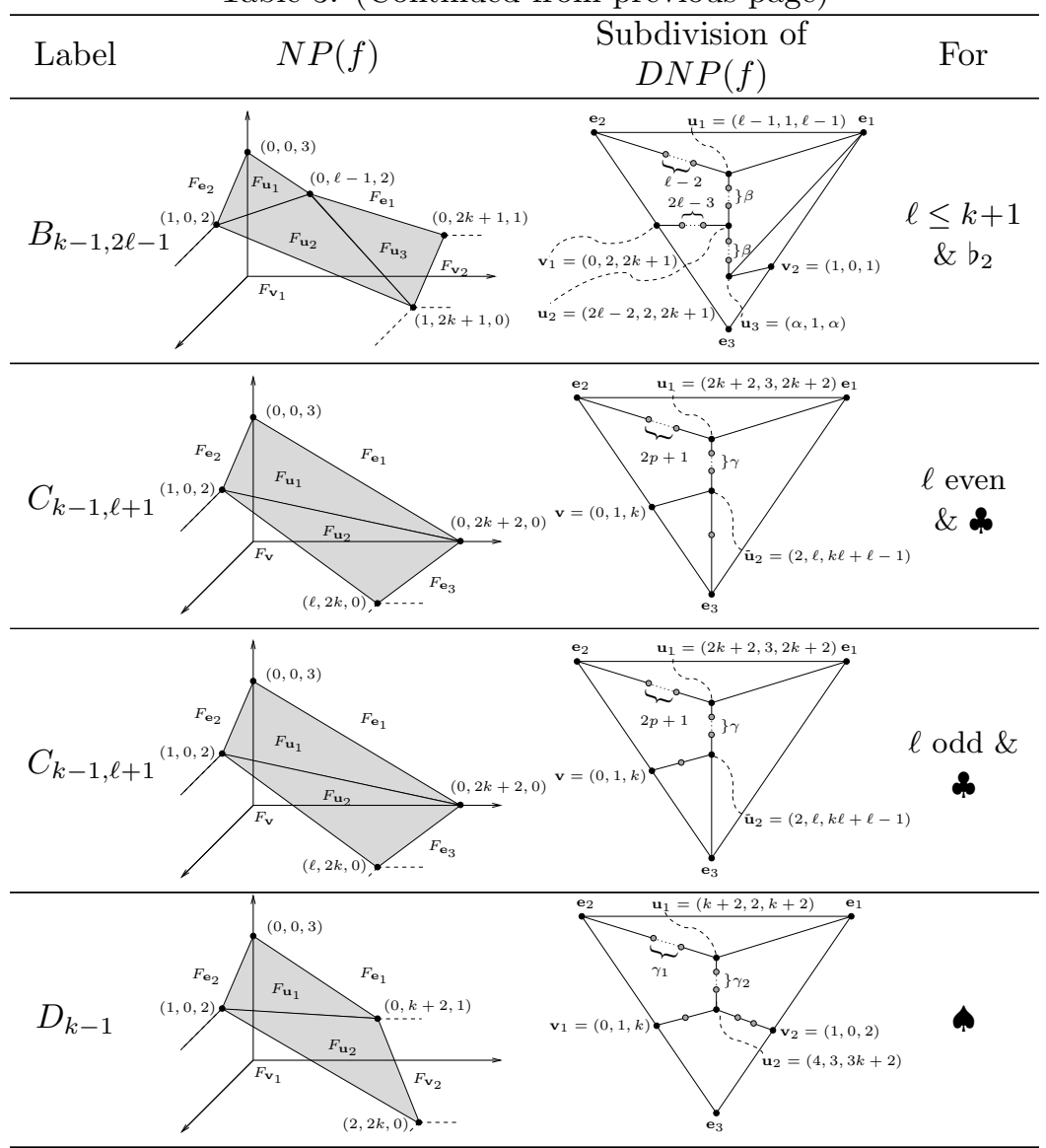

$b_{2}: \alpha=2 k-\ell+2, \beta=k-\ell+1$.

\{: $\gamma=p+\ell-1$ if $k=3 p+1 ; \gamma=p+\ell-2$ if $k=3 p+2$ or $k=3 p \cdot \gamma=\ell-2$ if $k=1$.

\$: $\gamma_{1}=\gamma_{2}=p$ if $k=2 p ; \gamma_{1}=\gamma_{2}=p+1$ if $k=2 p+1 . \gamma_{1}=1$ and $\gamma_{2}=0$ if $k=1$. 
Table 3. (Continued from previous page)

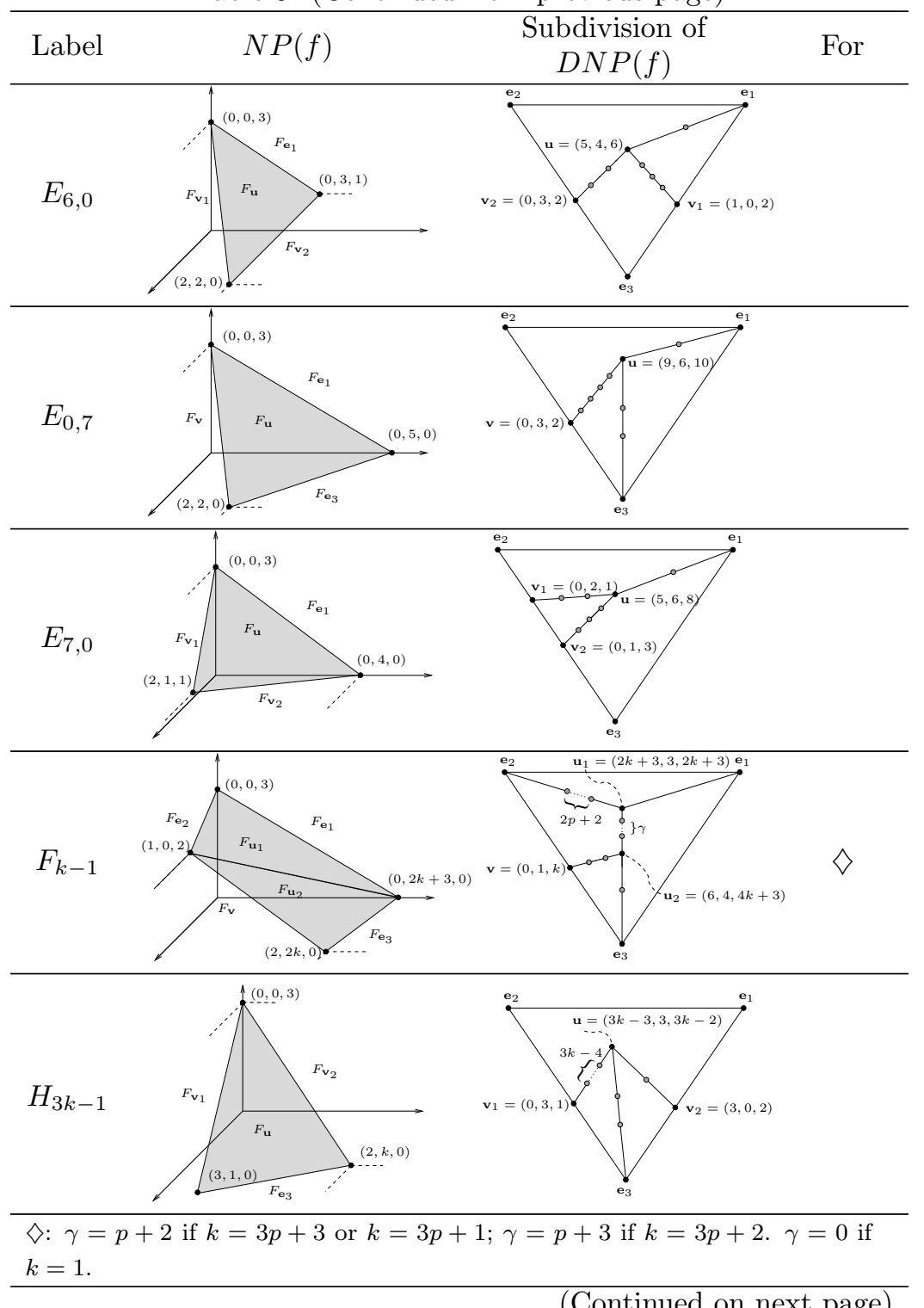

(Continued on next page) 
Table 3. (Continued from previous page)

Label $N P(f) \quad$ Subdivision of $c$

Appendix A. Here we list the diffeomorphisms we apply (in the given order) to transform Tjurina's matrix form of RTP-singularities into Miranda's given by equation (3.6).

$$
\begin{aligned}
& A_{k-1, \ell-1, m-1}: \text { (1) }(x, y, z, w) \longmapsto\left(x, y, z, w-y^{\ell}\right) \\
& \text { (2) }(x, y, z, w) \longmapsto(x-z-w, y, z, w) \\
& \text { (3) }(x, y, z, w) \longmapsto\left(x, y, z, w+x+y^{k}+z\right) \\
& B_{k-1,2 \ell}: \quad \text { (1) }(x, y, z, w) \longmapsto(x-z, y, z, w) \\
& B_{k-1,2 \ell-1}: \quad \text { (1) }(x, y, z, w) \longmapsto\left(x-z+y^{\ell-1}, y, z, w\right) \\
& C_{k-1, \ell+1}: \quad \text { (1) }(x, y, z, w) \longmapsto(x-z, y, z, w) \\
& \text { (2) }(x, y, z, w) \longmapsto\left(x, y, z, w-\sum_{i=1}^{\ell}\left(\begin{array}{l}
\ell \\
i
\end{array}\right) x^{\ell-i} z^{i-1}\right) \\
& \text { (3) }(x, y, z, w) \longmapsto(x, y, z, \alpha w)
\end{aligned}
$$




$$
\begin{aligned}
& \text { with } \alpha=1-\left(\begin{array}{l}
\ell \\
3
\end{array}\right) x^{\ell-3} y^{2 k} \\
& D_{k-1} \text { and } F_{k-1} \text { : } \\
& \text { (1) }(x, y, z, w) \longmapsto(x-z, y, z, w) \\
& \text { (2) }(x, y, z, w) \longmapsto\left(x, y, z, w-2 x y^{k}-y^{k} z\right) \\
& H_{3 k+1}: \\
& \text { (1) }(x, y, z, w) \longmapsto\left(x, y, z, w+y^{k}\right) \\
& E_{6,0}: \\
& \text { (1) }(x, y, z, w) \longmapsto\left(x, y, z, w+y^{2}\right) \\
& E_{7,0} \text { : } \\
& \text { (1) }(x, y, z, w) \longmapsto\left(x, y, z, w+x^{2}\right) \text {. }
\end{aligned}
$$

Note that additional row and column operations may be needed in some of the cases.

Acknowledgments. The authors would like to thank the referee for his/her careful reading and constructive comments.

\section{REFERENCES}

1. F. Aroca, M. Gómez-Morales and K. Shabbir, Torical modification of Newton non-degenerate ideals, preprint, arXiv:1209.5104.

2. M. Artin, On isolated rational singularities of surfaces, Amer. J. Math. 88 (1966), 129-136.

3. W. Barth, C. Peter and A. Van de Ven, Compact complex surfaces, SpringerVerlag, New York, 1984.

4. R. Bondil and D.T. Lê, Résolution des singularités de surfaces par éclatements normalisés (multiplicté, multiplicité polaire, et singularités minimales), Trends Math., Birkhauser, Basel, 2002.

5. Z. Chen, R. Du, S.-L. Tan and F. Yu, Cubic equations of rational triple points of dimension two, in American Mathematical Society, Providence, RI, 2007, 63-76.

6. T. de Jong and D. van Straten, A deformation theory for nonisolated singularities, Abh. Math. Sem. Univ. Hamburg 60 (1990), 177-208.

7. W. Decker, G.-M. Greuel, G. Pfister, and H. Schönemann, Singular 3-1-3-A computer algebra system for polynomial computations, Kaiserslautern, Germany, 2011, available at http://www.singular.uni-kl.de.

8. G. Ewald, Combinatorial convexity and algebraic geometry, Grad. Texts Math. 168, Springer Verlag, Berlin, 1996.

9. G. Ficher, Complex analytic geometry, Lect. Notes Math. 538, SpringerVerlag, Berlin, 1976. 
10. W. Fulton, Introduction to toric varieties, Princeton University Press, Princeton, 1993.

11. H. Grauert, Uber Modifikationen und exzeptionelle analytische Mengen, Math. Ann. 146 (1962), 331-368.

12. R. Hartshorne, Algebraic geometry, Grad. Texts Math. 52, Springer, New York, 1977.

13. H. Hironaka, Resolution of singularities of an algebraic variety over a field of characteristic zero I-II, Ann. Math. 79 (1964), 109-203: ibid. 79 (1964), 205326.

14. A.N. Jensen, Gfan, A software system for Gröbner fans and tropical varieties, 2009, Aarhus, Denmark, available at http://home.imf.au.dk/ jensen/software/gfan/gfan.html.

15. H.B. Laufer, Normal two-dimensional singularities, Ann. Math. Stud. 71, Princeton University Press, Princeton, N.J., 1971.

16. _ On rational singularities, Amer. J. Math. 94 (1972), 597-608.

17. _ Taut two-dimensional singularities, Math. Ann. 205 (1973), 131164.

18. D.T. Lê and B. Teissier, Variêtês polaires locales et classes de Chern des variêtês singuliêres, Ann. Math. 114 (1981), 457-491.

19. D.T. Lê and M. Tosun, Combinatorics of rational singularities, Comm. Math. Helv. 79 (2004), 582-604.

20. R. Miranda, Triple covers in algebraic geometry, Amer. J. Math. 107 (1985), 1123-1158.

21. D. Mond, Some remarks on the geometry and classification of germs of maps from surfaces to 3-spaces, Topology 26 (1987), 361-383.

22. D. Mumford, Algebaric geometry I: Complex projective varieties, Springer Verlag, New York, 1976.

23. Z. Oer, A. Özkan and M. Tosun, On the classification of rational singularities of surfaces, Int. J. Pure Appl. Math. 41 (2007), 85-110.

24. M. Oka, Non-degenerate complete intersection singularity, in Actualites mathematiques, Hermann, Paris, 1997.

25. J. Stevens, Partial resolutions of rational quadruple points, Inter. J. Math. 2 (1991), 205-221.

26. On the classification of rational surface singularities, preprint, arXiv: 1204.0269.

27. S.-L. Tan, Triple covers on smooth algebraic varieties, in Geometry and nonlinear partial differential equations, American Mathematical Society and International Press, 2002.

28. G.N. Tyurina, Absolute isolatedness of rational singularities and rational triple points, Fonc. Anal. Appl. 2 (1968), 324-332. 
29. M. Tosun, Tyurina components and rational cycles for rational singularities, Turkish J. Math. 23 (1999), 361-374.

30. A.N. Varchenko, Zeta-function of monodromy and Newton's diagram, Invent. Math. 37 (1976), 253-262.

31. J.M. Wahl, Equations defining rational singularities, Ann. Sci. Ecol. Norm. 10 (1977), 231-264.

32. O. Zariski, Polynomial ideals defined by infinitely near base points, Amer. J. Math. 60 (1938), 151-204.

Department of Mathematics, Yildiz Technical University, 34210, Istanbul, Turkey and Institute of Mathematical Sciences, Stony Brook University, STONY BRoOK, NY 11794

Email address: aysealtintas@gmail.com

Department of Mathematics, KoÇ University, Sariyer 34450, Istanbul, TURKEY

Email address: gulencevik@gmail.com

Department of Mathematics, Galatasaray University, Ortaköy 34357 , ISTANBUL, TURKEY

Email address: mtosun@gsu.edu.tr 